\title{
Evidências científicas sobre os impactos da parentabilidade e da influência mídiática no comportamento alimentar infantil
}

\author{
Scientific evidence on the impacts of parenting and media influence on children's eating behavior \\ Evidencia científica sobre los impactos de la crianza de los hijos y la influencia de los medios en la \\ conducta alimentaria de los niños
}

Recebido: 25/10/2021 | Revisado: 04/11/2021 | Aceito: 06/11/2021 | Publicado: 10/11/2021

\author{
Joyce Lopes Machado \\ ORCID: https://orcid.org/0000-0001-9544-3442 \\ Centro Universitário Santo Agostinho, Brasil \\ E-mail: joycelopessm@outlook.com \\ Deborah Laís Carvalho Santos \\ ORCID: https://orcid.org/0000-0002-4446-0127 \\ Centro Universitário Santo Agostinho, Brasil \\ E-mail: deborahlaisc@gmail.com \\ Tatiele Castelo de Oliveira \\ ORCID: https://orcid.org/0000-0002-1240-9140 \\ Centro Universitário Santo Agostinho, Brasil \\ E-mail:oliveirac.tatiele@gmail.com \\ Liejy Agnes dos Santos Raposo Landim \\ ORCID: https://orcid.org/0000-0002-8214-2832 \\ Centro Universitário Santo Agostinho, Brasil \\ E-mail: liejyagnes@gmail.com
}

\begin{abstract}
Resumo
Introdução: As propagandas de produtos influenciam a formação de novos consumidores, utilizando-se da mobilização de suas fantasias, forjando uma sensação de felicidade ao adquirir determinado produto, se tornando fundamental no processo de convencimento dos pais, que são os encarregados pela compra.Objetivo:Analisar as evidências científicas da relação parental e da influência midiática sobre o comportamento alimentar infantil. Metodologia: Trata-se de uma de revisão narrativa da literatura, com abordagem teórica. A busca dos artigos foi realizada nas seguintes bases de dados eletrônicas: Scientific Electronic Library Online (SciELO), National Library of Medicine (PubMED) e Literatura Latino-Americana e do Caribe de Informações em Ciências da Saúde (LILACS), através dos descritores em português e inglês: "Comportamento alimentar", "criança", "alimentos", devidamente cadastrados nos Descritores em Ciência da Saúde (DeCS) num recorte temporal proposto, entre os anos de 2011 a 2021. Resultados: A pesquisa foi subdividida em duas categorias que compuseram o corpo discursivo do texto: Relação parental e o comportamento alimentar em crianças e Implicações da mídia no comportamento alimentar infantil. Considerações finais: Portanto, a parentabilidade e a influência da mídia, apresentam grande impacto na vida das crianças, que estão em processo de aprendizagem e se tornam mais frágeis em relação a esses fatores.
\end{abstract}

Palavras-chave: Comportamento alimentar; Criança; Alimentos.

\begin{abstract}
Introduction: Product advertisements influence the formation of new consumers, using the mobilization of their fantasies, forging a feeling of happiness when purchasing a certain product, becoming essential in the process of convincing parents, who are responsible for the purchase. Goal:To analyze the scientific evidence of parental relationship and media influence on children's eating behavior. Methodology: This is a narrative literature review with a theoretical approach. The search for articles was performed in the following electronic databases: Scientific Electronic Library Online (SciELO), National Library of Medicine (PubMED) and Latin American and Caribbean Literature on Health Sciences Information (LILACS), using the descriptors in Portuguese and English: "Eating behavior", "child", "food", duly registered in the Health Science Descriptors (DeCS) in a proposed time frame, between the years 2011 to 2021. Results: The research was subdivided into two categories which made up the discursive body of the text: Parental relationship and eating behavior in children and Implications of the media in children's eating behavior. Final considerations: Therefore, parenting and the influence of the media have a great impact on the lives of children, who are in the learning process and become more fragile in relation to these factors.
\end{abstract}

Keywords: Eating behavior; Child; Food. 


\begin{abstract}
Resumen
Introducción: Los anuncios de productos influyen en la formación de nuevos consumidores, utilizando la movilización de sus fantasías, forjando un sentimiento de felicidad al comprar un determinado producto, volviéndose imprescindible en el proceso de convencer a los padres, que son los responsables de la compra.: Analizar lo científico evidencia de la relación de los padres y la influencia de los medios en la conducta alimentaria de los niños. Metodología: Se trata de una revisión de literatura narrativa con enfoque teórico. La búsqueda de artículos se realizó en las siguientes bases de datos electrónicas: Scientific Electronic Library Online (SciELO), National Library of Medicine (PubMED) y Literatura Latinoamericana y del Caribe sobre Información en Ciencias de la Salud (LILACS), utilizando los descriptores en portugués e inglés: “ Comportamiento alimenticio "," niño "," alimentación ", debidamente registrados en los Descriptores de Ciencias de la Salud (DeCS) en un marco temporal propuesto, entre los años 2011 a 2021. Resultados: La investigación se subdividió en dos categorías que conformaron el cuerpo discursivo del texto: Relación parental y conducta alimentaria en niños e Implicaciones de los medios de comunicación en la conducta alimentaria infantil. Consideraciones finales: Por tanto, la paternidad y la influencia de los medios de comunicación tienen un gran impacto en la vida de los niños, que se encuentran en proceso de aprendizaje y se vuelven más frágiles en relación a estos factores.
\end{abstract}

Palabras clave: Comportamiento alimentício; Niño; Comida.

\title{
1. Introdução
}

A publicidade está presente em diversos momentos do nosso cotidiano, pois somos afetados continuamente, durante toda a vida, por anúncios de produtos e serviços que podem ser capazes de satisfazer nossas necessidades e mexer com os nossos desejos. No caso do público infantil, as propagandas de produtos alimentícios têm como propósito a influência e formação de novos consumidores e, para isso, utiliza-se da mobilização de suas fantasias, forjando uma sensação de felicidade ao adquirir determinado produto, o que se torna fundamental no processo de convencimento dos pais, que são os encarregados pela compra (Melo et al.,2019).

Essa influência, que tem sido fortemente direcionada à população infantil, nem sempre acarreta efeitos positivos, uma vez que, a disseminação de alimentos considerados não saudáveis e a indução à sua compra podem causar sérios problemas à saúde das crianças, podendo assim, ocasionar prejuízos a esta parte da população (Peternella; Gomes, 2019).

Por isso, acredita-se que a melhor maneira de reduzir esses indícios ainda é por meio da prevenção, educando as crianças para a prática de hábitos alimentares saudáveis e através da conscientização dos pais, a fim de que sejam verdadeiros espelhos para seus filhos, visto que, é notório que as práticas parentais estão intresicamente ligadas ao comportamento alimentar infantil (Berleze et al., 2020).

A questão do marketing, em suas diversas formas, coopera na persuasão das crianças no consumo de determinados alimentos e é de fato preocupante (Moura, 2015). A exposição ao público infantil destaca que alimentos calóricos e ultraprocessados têm contribuído para a epidemia da obesidade, além de trabalhar a lembrança e familiaridade dos alimentos e marcas, que forma uma combinação positiva com os produtos, incentivando e influenciando a compra e consumo de tais (Kelly et al., 2016). Dessa forma, os fast foods pretendem que suas marcas estejam sempre em evidência, tendo como consequência, o aumento de ingestão de seus produtos, sem considerar o valor nutritivo e os danos da ingestão excessiva à saúde do consumidor. (Milane et al., 2015).

Nos últimos 30 anos, pesquisas realizadas em âmbito nacional apontam que há um aumento significativo nas taxas de sobrepeso e obesidade infantil em todas as regiões do Brasil. A mídia e as propagandas de alimentos possuem estratégias capazes de influenciar a promoção e venda de produtos de forma a moldar a conduta de compra do consumidor. Observa-se que a sociedade contemporânea sofreu uma transição nutricional, onde se parte de uma dieta minimamente processada para uma dieta rica em açúcares, óleos e gorduras. Com isto, a predominância de obesidade tem crescido significativamente no mundo e especialmente na infância (Carvalho, 2019). 
Diante do exposto, o presente estudo visa analisar as evidências científicas da relação parental e da influência midiática sobre o comportamento alimentar infantil.

\section{Metodologia}

Trata-se de uma de revisão narrativa da literatura, com abordagem teórica, que propõe como resultados informações referentes à influência da relação parental e midiática sobre o comportamento alimentar infantil. Os artigos de revisão narrativa têm por objetivo mapear o conhecimento sobre uma questão ampla para descrever e discutir o desenvolvimento ou o estado da arte de um determinado assunto, sob ponto de vista teórico ou contextual (Martins, 2018).

A busca dos artigos foi realizada nas seguintes bases de dados eletrônicas: Scientific Electronic Library Online (SciELO), National Library of Medicine (PubMED) e Literatura Latino-Americana e do Caribe de Informações em Ciências da Saúde (LILACS).Para o levantamento dos estudos foram utilizados os seguintes descritores, em português: "Comportamento alimentar", "criança", "alimentos" e em inglês: "Eating behavior", "child", "food", no qual, a fim de propiciar uma maior captura de estudos foi realizado o cruzamento entre os descritores supracitados, devidamente cadastrados nos Descritores em Ciência da Saúde (DeCS).

Para a construção desta revisão narrativa foram escolhidos os seguintes critérios de inclusão: artigos científicos originais que constituíam a temática do estudo, idiomas português e inglês, publicações indexadas no recorte temporal proposto, entre os anos de 2011 a 2021. Por conseguinte, os critérios de exclusão foram: artigos de revisão, teses, dissertações, artigos originais publicados fora da temporalidade indicada, pesquisas experimentais com animais e estudos que se repetiam nas bases eletrônicas.

A pesquisa foi subdividida em duas categorias para a realização do desenvolvimento do estudo, sendo elas: Relação parental e o comportamento alimentar em crianças e Implicações da mídia no comportamento alimentar infantil.

\section{Resultados e Discussão}

\section{Relação parental e o comportamento alimentar em crianças}

Segundo, Dantas; Silva (2019), a infância é o período que se faz muito importante na vida de cada pessoa, pois consiste em um momento em que as influências recebidas do ambiente no qual está inserida se fazem relevantes no processo de construção de hábitos e comportamentos que serão adquiridos para toda a vida adulta. Nesse sentido, Birch (2011) afirma que está claro que, fatores socioculturais, econômicos e psicossociais fazem-se presentes, de maneira impactante nos hábitos alimentares infantis, admitindo assim, um papel fundamental no comportamento alimentar desde a primeira infância.

Aparício (2013), nesse seguimento, diz que a família caracteriza-se por ser a instituição educadora que mais induz a criança no que diz respeito ao perfil de vivência alimentar, embora atravesse uma fase de intensas transformações em seu paradigma. Desse modo, os pais admitem a função de educadores nutricionais por meio do convívio familiar, obtendo respaldo direto nas estratégias realizadas frente ao comportamento alimentar das crianças.

Huçalo e Ivatiuk (2017) em um estudo descritivo buscaram averiguar se existe influência direta no modelo alimentar das crianças devido ao padrão adquirido pelos pais. De acordo com os resultados, foi certificado o fato de que hábitos alimentares parentais favoráveis podem impactar positivamente no comportamento alimentar e no desenvolvimento da criança, visto que, o ambiente propicia uma relação satisfatória da mesma com os alimentos.

Um exemplo disto pode ser observado no estudo supracitado, onde se evidenciou que os filhos são naturalmente controlados pelos pais e que se estes oferecem uma conduta saudável, onde a alimentação será bem preparada e com todos os 
afetos parentais envolvidos, as crianças, por sua vez, tendem a aumentar cada vez mais o prazer pela comida, proporcionando um bom desenvolvimento e comportamento alimentar reforçador.

Entretanto, tal influência também pode ser negativa, como foi exposto no estudo de Melo; Cruz; Brito; Pinho (2017) onde o ambiente familiar foi intrinsecamente ligado ao excesso de peso infantil. Na avaliação do consumo alimentar das crianças desta investigação, boa parte delas realizavam suas refeições com os pais, em sua própria residência e tal distúrbio nutricional foi notificado devido ao consumo alimentar dominante de guloseimas e refeições datadas "especiais" pelos seus familiares.

Tal conduta pode ser explicada pelo processo de transição nutricional, onde se modificam alguns padrões, levandonos a uma predominância de alimentos hipercalóricos, ricos em açúcares, sódio e gorduras. Por fim, o comportamento alimentar dos pais, de certa forma, possibilitou alterações nos índices de massa corpórea dessas crianças, acarretando um aumento estatístico de sobrepeso e obesidade infantil entre elas.

Ferrer et al., (2017) em seu estudo afirmou que fatores emocionais dos pais estão diretamente ligados a alimentação emocional e que isso pode afetar nos hábitos alimentares das crianças, pois, quanto maior for a supressão emocional, maior o consumo hedônico (ato de comprar algo por impulso) e consequentemente maior a ingestão de alimentos de alta densidade calórica, implicando negativamente no comportamento alimentar das crianças por apego de tais alterações no processo regulatório emocional.

Em outro estudo, Persky e Yaremych (2021) realizaram uma pesquisa na qual foram avaliadas as atribuições genéticas dos pais em relação ao comportamento alimentar dos filhos. De todos os pontos, o que mais obtiveram destaque foram as competências direcionadas à baixa eficácia para alimentação das crianças em termos de quantidade correta e preferências alimentares ruins (alimentos de alto teor calórico), considerando, desse modo, que o intuito da genética sobre o comportamento alimentar infantil está associado com as relações afetivas e conduta desadaptativa dos pais em termos de alimentação e nutrição.

\section{Implicações da mídia no comportamento alimentar infantil}

De acordo com Lanes et al., (2021), a mídia pode ser considerada um importante agente influenciador do comportamento alimentar infantil, pois estabelece padrões mediante a forma de como a informação é difundida. Hartung; Karageorgiadis (2017). Nesse contexto, o marketing tem crescido cada vez mais, em diferentes variações, como anúncios impressos, comerciais televisivos, spots de rádio, banners na internet, embalagens, promoções, dentre outros, buscando sempre inovar suas estratégias, a fim de adentrar, de forma imanente, o cotidiano de todas as pessoas, especialmente das crianças, devido a sua vulnerabilidade.

Desse modo, Theodoro (2018) explica que já é reconhecido algumas práticas parecem afetar diretamente a conceituação do que é um "bom alimento" pelas crianças, que acabam entendendo que tal designação caracteriza-se apenas pela sua aparência, sabor doce, textura e diversão .Nessa perspectiva, Magalhães et al., (2020) afirma que a propaganda de alimentos destinada ao público infantil está relacionada à presença de produtos altamente processados, ligados com personagens infantis, brindes e a exposição a diversas modalidades, que durante a refeição são obstáculos que rodeiam a vida dos familiares.

Nessa perspectiva, Henriques, Sally e Beiler; (2012) afirma em seu estudo, o quanto a indústria alimentícia enxerga a criança como indivíduo consumidor e o quanto este fator está cada vez mais ligado ao fato desta ter grande prestígio na escolha de compra dos pais, devido ao seu considerável poder de convencimento, visto que, as crianças influenciam de forma evidente na decisão das compras de gêneros alimentícios da família, chegando a um percentual de $92 \%$, o que faz com que as empresas renovem cada vez mais o marketing destinado ao público infantil. 
Diante disso, segundo Nunes (2011), muitos pais aplicam estratégias autoritárias e/ou flexíveis no acesso á comida, podendo também promover a preferência paradoxal das crianças por alimentos hipercalóricos. Isto é, os pais podem incentivar o gosto das crianças por insumos energeticamente densos ao restringir o acesso aos mesmos na tentativa de usá-los para moldar o comportamento dos filhos, por conseguinte, sendo associados como prêmio por um comportamento positivo.

No estudo transversal de Santos et al., (2012) foi feita uma análise com duas emissoras brasileiras da televisão aberta, que buscou verificar a quantidade de propagandas sobre produtos alimentícios e o respectivo horário pelas quais eram transmitidas na TV. Os dados foram coletados através de gravações da programação das emissoras, durante 28 dias, nos horários da manhã, tarde e noite.

Foi constatado, no estudo acima, que o horário predominante das divulgações comerciais era de 08 às 12 horas, o que dessa forma, resulta que a intencionalidade do horário sugere captar o público infantil, visto que caracteriza um intervalo propício para essa faixa etária. Em relação as propagandas, $85 \%$ anunciavam produtos contendo açúcar, óleo e gordura, não sendo identificado nenhum comercial que abordasse o consumo de frutas, verduras ou hortaliças, levando essas crianças a se atraírem fortemente por esses alimentos de baixa qualidade nutricional.

Concomitante a isto, Smit et al., (2020) objetivou investigar a repercussão dos vlogs no comportamento alimentar das crianças, em seu achado longitudinal. As análises evidenciaram que o número de crianças que assistiram os vlogs e a frequência de consumo de bebidas e lanches não saudáveis aumentou de forma proporcional e obtivera efeito significativo, devido à descoberta das formas mais impactantes do marketing de alimentos.

Em um teste aleatório, Coates et al., (2019) realizou um projeto com 176 crianças, que foram selecionadas para observar dois perfis distintos de influenciadores (um mostrava imagens de alimentos saudáveis e o outro alimentos não saudáveis). As crianças que viram o influenciador com lanches não saudáveis elevaram consideravelmente a ingestão desses alimentos em comparação com as crianças que viram o influenciador com lanches saudáveis.

Ainda nesse enquadramento, Norman et al., (2018) em seu estudo intra-sujeito randomizado, realizou um tipo de publicidade online em dois momentos: antes do lanche e depois do almoço. Após a exposição de cada conteúdo, foi calculado a ingestão alimentar das crianças em quilojoules. (KJ). Concluiu-se que todas as crianças comeram mais logo após as exposições publicitárias referentes a alimentos, o que resultou num aumento de $182 \mathrm{KJ}$ em relação à ingestão calórica diária. Ademais, a publicidade online obteve impacto maior entre crianças com sobrepeso, em ambos os grupos de mídia.

Nesse contexto, mediante Karageorgiadis (2014), é sabido que, de acordo com o Estatuto da Criança e do Adolescente (ECA - Lei 8.069/90) e também do Código de Defesa do Consumidor, essas estratégias de comunicação mercadológicas dispostas ao público infantil são ilegais, ainda que sejam muito frequentes e que o público infantil é o mais susceptível aos apelos das mídias sociais, por não possuir maturidade suficiente para discernir entre o bom e o ruim.

Dessa forma, Prodanov e Cimadon (2016) notifica que cabe ainda mais esforços dos profissionais da saúde e da família para impedir a expansão do marketing de anúncios referentes a alimentos industrializados com apelo infantil, com o intuito de conscientização sobre a importância da adoção de hábitos de vida saudáveis e de uma alimentação que promova o crescimento e o desenvolvimento das crianças, bem como a promoção da saúde.

\section{Considerações Finais}

Em síntese, está claro que, a parentabilidade e a influência da mídia de modo geral, apresentam grande impacto na vida das pessoas, especialmente, das crianças, que estão em processo de aprendizagem e se tornam mais frágeis em relação a esses fatores. Nesse sentido, é evidente que se torna fundamental o acompanhamento incansável da família na execução de 
práticas educativas positivas com o intuito de estimular hábitos alimentares saudáveis adequados que venham ser adquiridos pelas crianças.

Além disso, faz-se necessário fomentar a disseminação de conteúdos sobre a promoção de alimentos saudáveis nas redes sociais, a fim de impulsionar a adoção de comportamentos alimentares benéficos ao público infantil, que supram suas necessidades energéticas, para que sua saúde seja preservada e assim contribua para o bom andamento da sociedade. Por fim, mais estudos sobre o reflexo do comportamento dos pais em relação à conduta alimentar dos filhos, bem como estratégias de educação alimentar e nutricional para ao público infantil, orientações sobre decisões de compra, são indispensáveis para a compreensão dos efeitos da relação parental e do marketing de alimentos sobre as crianças e também para informar as intervenções sócio-políticas apropriadas.

\section{Referências}

De Melo, J. D. C. B., Lustoza, G. F., Ibiapina, D. F. N., \& Landim, L. A. D. S. R. (2019). Influência da mídia no consumo de alimentos ultraprocessados e no estado nutricional de escolares. Revista Eletrônica Acervo Saúde, (29), e1016-e1016.

Veltrini, C. P., \& da Gomes Ribeiro, C. (2019). Publicidad, obesidad infantil y violación del derecho humano a una alimentación adecuada: un análisis a la luz de la bioética. Revista Iberoamericana de Bioética, (9), 1-14.

Berleze, K. J., Tomasi, B., Medeiros, L. B., Artifon, M., \& Brito, A. C. (2020). Influência do Comportamento Parental na Obesidade Infantil: Revisão da Literatura. Revista Pleiade, 14(31), 106-113.

Moura, N. C de. (2016). Influência da mídia no comportamento alimentar de crianças e adolescentes. Segurança Alimentar e Nutricional.Campinas, SP. 17(1): 113-122.

Kelly B. et al. (2016). Televisionadvertising, notviewing, isassociatedwith negative dietarypatterns in children. Pediatricobesity. 11(2), 158-160.

Milane M. O. et al. (2015). Influência da mídia nos hábitos alimentares de crianças: uma revisão da literatura. 5(3);1-5.

Carvalho G. R (2019). Percepção sobre mídia e comportamento na compra de alimentos: estudo com consumidores de dois municípios do sul de Minas Gerais. Braz. J. FoodTechnol. Campinas. 22:1-8.

Martins M. D. F. M. (2018). Estudos de revisão de literatura. Rio de Janeiro: FIOCRUZ/ICICT:37

Dantas R., \& Silva G. (2019). The role of the obesogenic environment and parental lifestyle in infant feeding behavior. Revista Paulista de Pediatria, São Paulo.37(3):363-371.

Birch L. L. et al. (2011). Confirmatory factor analysis of the Child Feeding Questionnaire: A measure of parental attitudes, beliefs and practices about child feeding and obesity proneness. Appetite. 36, 201-210.

Aparícia G. et al. (2013). Nutritional status in preschool children: current trends of mother's body perception and concerns. Atención Primaria. 45(2); 194200 .

Huçalo A. P., \& Ivatiuk A. L. (2017). A relação entre práticas parentais e o comportamento alimentar em crianças. Revista PsicoFAE: Pluralidades em Saúde Mental.6 (2);113-128.

Melo K. M. et al. (2017). Influência do comportamento dos pais durante a refeição e no excesso de peso na infância. Escola Anna Nery. 21.

Ferrer R. A. et al. (2017). Supressão de emoções, comer emocional e comportamento alimentar entre díades pais-adolescentes. Emoção.17(7):1052.

Persky S., \&Yaremych H. E (2021). Genetic attributions of parents to children's eating behavior: relationships with beliefs, emotions and behavior in food choice. Apetite. 155:104824.

Lanes, D. V. C. (2021). Estratégias lúdicas para a construção de hábitos alimentares saudáveis na educação infantil. Revista Ciências \& Ideias. 4(1).: 21761477 .

Hartung P. A. D., \& Karageorgiadis. (2017). A regulação da publicidade de alimentos e bebidas não alcoólicas para crianças no Brasil. Revista de Direito Sanitário. 17(3):160-184.

Theodoro D. T. (2018). Avaliação do conteúdo de propagandas alimentícias para o público infantil em canais de televisão. Revista Ciências Nutricionais Online. 2(2): 47-52

Magalhães, M. R. et al. (2020). "Socorro! Meu Filho Come Mal”: uma análise do comportamento alimentar infantil. Perspectivas Em Análise Do Comportamento. 11(1): 079-091.

Henriques, P. et al. (2012). Regulation of children's food advertising as a health promotion strategy. Ciência \& Saúde Coletiva. 17(2):481-490. 
Research, Society and Development, v. 10, n. 14, e474101422240, 2021

(CC BY 4.0) | ISSN 2525-3409 | DOI: http://dx.doi.org/10.33448/rsd-v10i14.22240

Nunes, L. A. (2011). A influência dos estilos parentais na obesidade infantil. International Journal of Developmental and Educational Psychology: INFAD. Revista de Psicología. 1(1):37-46.

Santos, C. C. et al. (2012). The influence of television on eating habits, customs and behavior. Cogitare Enfermagem. 17(1): 65-71.

Smit, C. R et al. (2020). O impacto dos influenciadores das redes sociais no comportamento alimentar das crianças. Fronteiras em psicologia.10: 2975.

Coates, A. E et al. (2019). Social media influencer marketing and children's food intake: a randomized trial. Pediatrics.143.

Norman, J., et al. (2018).Sustained impact of energy-dense TV and online food advertising on children's food intake: an in-house, randomized, crossover, counterbalanced study. Jornal internacional de nutrição comportamental e atividade física. 15(1):1-11.

Karageorgiadis, E. (2014). Comunicação mercadológica abusiva dirigida à criança e prática de venda casada. Revista Luso-Brasileira de Direito do Consumo. 2(14):11.

Prodanov, S. S., Cimadon, H. M. S. (2016). A influência da publicidade nos hábitos alimentares de crianças em idade escolar. Revista conhecimento online. 